\title{
MYH3 is a novel gene associated with carotid paragangliomas
}

\author{
A. Snezhkina ${ }^{1 *}$, E. Lukyanova ${ }^{1}$, D. Kalinin ${ }^{2}$, E. Pudova ${ }^{1}$, M. Fedorova ${ }^{1}$, \\ S. Kharitonov ${ }^{1}$, Z. Guvatova ${ }^{1}$, I. Abramov ${ }^{1}$, G. Krasnov ${ }^{1}$, N. Melnikova ${ }^{1}$, \\ A. Dmitriev ${ }^{1}$, A. Kudryavtseva ${ }^{1,3}$ \\ ${ }^{1}$ Engelhardt Institute of Molecular Biology RAS, Moscow, Russia \\ ${ }^{2}$ Vishnevsky Institute of Surgery, Ministry of Health of the Russian Federation, Moscow, Russia \\ ${ }^{3}$ National Medical Research Radiological Center, Ministry of Health of the Russian Federation, \\ Moscow, Russia \\ *e-mail:leftger@rambler.ru
}

Key words: carotid paragangliomas, exome, mutations, MYH3, high-throughput sequencing

Motivation and Aim: Carotid paragangliomas are relatively rare tumors arising from the paraganglia of carotid body at the bifurcation of carotid arteries. Approximately $40 \%$ of paragangliomas are hereditary, and currently over 30 different genes have been associated with the disease. However, molecular genetic mechanisms underlying carcinogenesis of carotid paragangliomas are still not fully understood.

Methods and Algorithms: We have collected 52 carotid paragangliomas derived from Vishnevsky Institute of Surgery, Ministry of Health of the Russian Federation. The isolation of DNA and RNA from the samples was performed. Specialized Illumina kits (USA) were used for exome and transcriptome library preparation. Exome sequencing was performed in 2x75 bp paired-end model using NextSeq 500 System (Illumina) at EIMB RAS "Genome" center (http://www.eimb.ru/rus/ckp/ccu_genome_c.php). Sequencing of transcriptome libraries was carried out using HiSeq 4000 (Illumina) with 2x100 bp paired-end reads. Bioinformatic analysis of the data was performed using XSEQ software that allows computing the influence of somatic mutations on gene expression profile [1].

Results: Using XSEQ software, we analyzed the exome and transcriptome data from carotid paragangliomas. We found that mutations in $M Y H 3$ gene impact its expression and, therefore, can have potential phenotypic effect. Different mutations were observed in MYH3 gene including three probably pathogenic ones (NM_002470.3: p.Ile264Thr/ c.791T $>$ C (rs763347751, chr17: 10550688), NM_002470.3: p.Ala1752Thr/c.5254G $>$ A (rs34393601, chr17: 10534960), and NM_002470.3: p.Ala1604Thr/c.4810G>A (rs201488879, chr17: 10535939), according to Sift and PolyPhen 2 prediction tools. These mutations can change the structure of the protein and, therefore, affect its function. Conclusion: A novel gene, MYH3, which can be involved in carcinogenesis of carotid paragangliomas, was identified.

Acknowledgements: This work was funded by the Russian Science Foundation, grant 17-75-20105.

\section{References}

1. Ding J. et al. (2015) Systematic analysis of somatic mutations impacting gene expression in 12 tumour types. Nat. Commun. 6:8554. 